\title{
Functioning Metastases from Thyroid Papillary Carcinoma in Bone
}

Abdelhamid Biyi, Soukaina Zaïmi, and Abderrahim Doudouh

Department of Nuclear Medicine, Mohammed V Military Teaching Hospital, Mohammed V University of Rabat, Rabat, Morocco

Functioning thyroid metastases are a rare cause of hyperthyroidism. Most are follicular carcinoma. Here, we report a case in which a 62-y-old man with a history of right subtotal thyroidectomy for a benign adenoma complained of symptoms of hyperthyroidism associated with left arm pain. Biopsy of a humeral lesion was consistent with papillary carcinoma metastatic from the thyroid. Postoperatively, the patient received a cumulative dose of $14.8 \mathrm{GBq}$ of ${ }^{131}$ I with good control of the hyperthyroidism but without eradication of the bone metastasis.

Key Words: thyrotoxicosis; papillary thyroid carcinoma; functioning metastases; SPECT-CT

J Nucl Med Technol 2016; 44:253-254

DOI: 10.2967/jnmt.116.174573

$\mathbf{F}$

unctioning thyroid metastases are a rare cause of hyperthyroidism. Fewer than 100 cases can be found in the worldwide literature. Most of them were follicular carcinoma with lung or bone involvement. Only 20 cases of functioning metastases from papillary carcinoma have been reported $(1,2)$.

\section{CASE REPORT}

A 62-y-old man who had undergone right subtotal thyroidectomy 11 y previously for a benign adenoma had recently presented with symptoms of hyperthyroidism associated with left arm pain. His level of free thyroxine was elevated (68 pmol/L; reference level, 11-25 pmol/L), his level of thyroid-stimulating hormone (TSH) was suppressed $(<0.01 \mu \mathrm{IU} / \mathrm{mL}$; reference level, 0.2-3.2 $\mu \mathrm{IU} / \mathrm{mL})$, and he was negative for TSH-receptor antibody. ${ }^{99 \mathrm{~m} T c-s o d i u m ~ p e r t e c h-~}$ netate planar scintigraphy of the neck and upper chest showed mild uptake in the left thyroid lobe, in contrast to heightened uptake in the left arm (Fig. 1). The planar posterior view and SPECT/CT fusion images showed focal, intense uptake in the fifth thoracic vertebra (Fig. 2), an area of involvement that had been asymptomatic at the first presentation but had become symptomatic within about $50 \mathrm{~d}$. ${ }^{99 \mathrm{~m}} \mathrm{Tc}$-methylendiphosphonate

\footnotetext{
Received Feb. 20, 2016; revision accepted May 10, 2016.

For correspondence or reprints contact: Abdelhamid Biyi, P.O. Box 6614, Rabat Instituts, Rabat 10101, Morocco.

E-mail: abdelhamidbiyi@yahoo.fr

Published online Jun. 30, 2016.

COPYRIGHT (c) 2016 by the Society of Nuclear Medicine and Molecular Imaging, Inc.
}

bone scanning showed a focus of increased uptake in the left humerus and a photopenic area in the right pelvic bone (Fig. 3). No other sites of bone involvement were detected. Biopsy of the left humeral lesion was consistent with papillary carcinoma metastatic from the thyroid. Left subtotal thyroidectomy was therefore performed, but no malignancy was found in the remaining lobe. The clinical course was marked by a rapid onset of medullar compression symptoms. Decompressive laminectomy was recommended, but the patient refused to undergo more surgery. Despite a persistently low TSH level, postablation ${ }^{131} \mathrm{I}$ whole-body scans showed bone metastases (Fig. 4). Six months after the patient had completed radioiodine therapy (3.7 GBq), the myelopathic symptoms improved but free thyroxine and triiodothyronine remained elevated. In the 48th mo after the diagnosis, he finally received a cumulative dose of $14.8 \mathrm{GBq}$ of ${ }^{131} \mathrm{I}$, which controlled the hyperthyroidism well but did not eradicate the bone metastases. Antithyroid drugs were replaced with lifelong TSH suppression.

\section{DISCUSSION}

Three criteria on which to base the diagnosis of functioning metastases were present in our patient: failure of thyrotoxicosis to resolve after thyroidectomy, mild iodine uptake in the remnant thyroid lobe, and iodine uptake in metastases (2). Furthermore, there was an unusual preoperative accumulation of ${ }^{99 \mathrm{~m}}$ Tc-pertechnetate in functioning metastases from thyroid papillary carcinoma in bone along with a low TSH level caused by thyrotoxicosis. These metastases, although readily visible with ${ }^{131} \mathrm{I}$, were lost among the normal skeletal uptake when imaged using ${ }^{99 \mathrm{~m}} \mathrm{Tc}$-methylendiphosphonate because of the absence of osteoblastic reaction (3). One recent publication

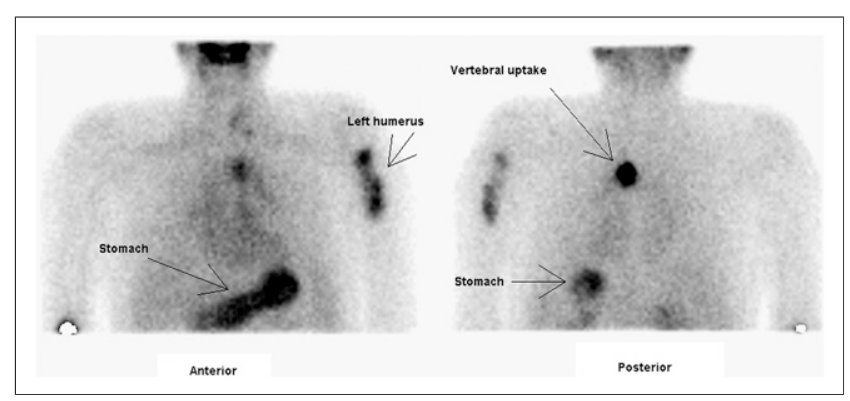

FIGURE 1. Mild uptake in left thyroid lobe on images obtained using ${ }^{99 m}$ Tc-sodium pertechnetate, in contrast to heightened uptake in left arm. Posterior view shows focal, intense uptake in posteromedial region. 
(4) involving 54 patients suggested that there is $100 \%$ agreement between ${ }^{99 \mathrm{~m}} \mathrm{Tc}$-pertechnetate scans and iodine scans in the evaluation of bone metastases from thyroid cancer. Given

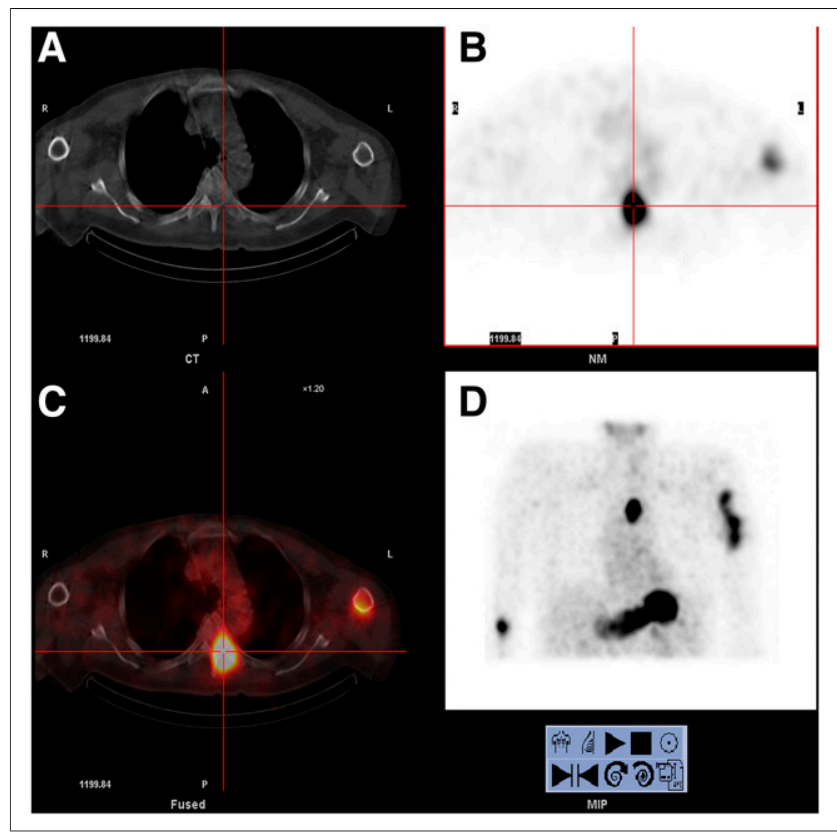

FIGURE 2. Transaxial CT image (A), SPECT image (B), fusion SPECT/CT image (C), and maximum-intensity projection (D) showing intense uptake at lytic area at left lamina of fifth thoracic vertebra.

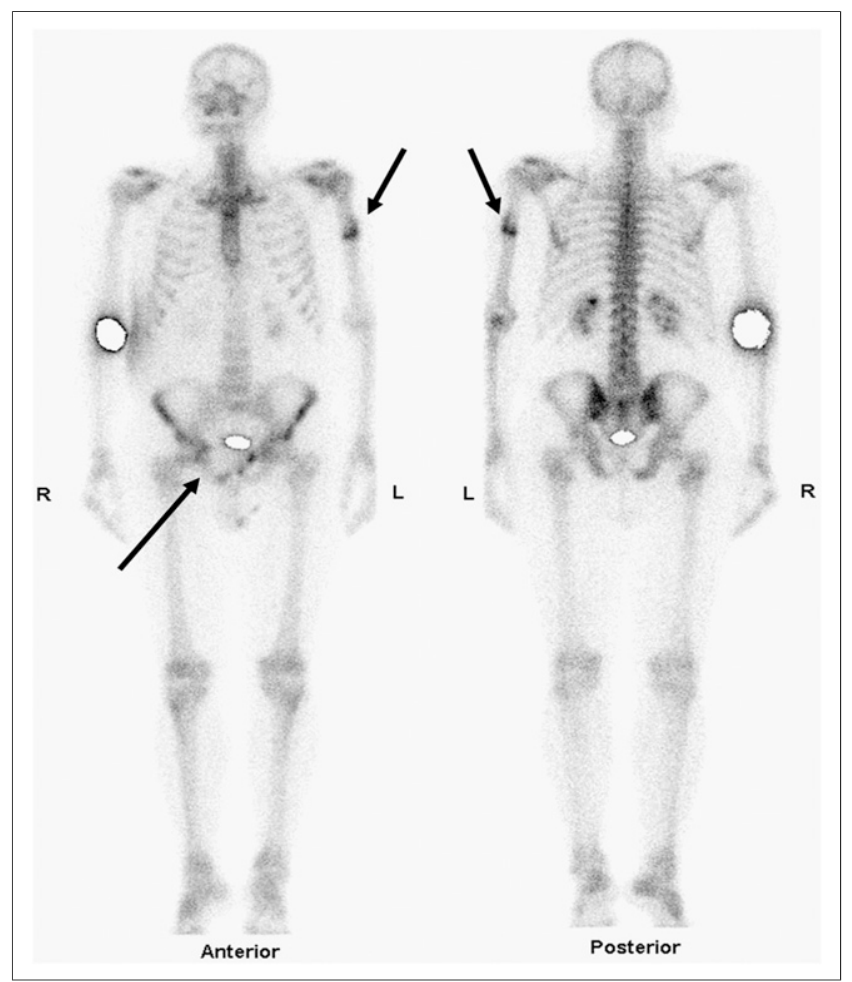

FIGURE 3. Bone scan showing focus of increased uptake in left humerus (upper arrows) and photopenic area in right bone of pelvis (lower arrow). Injection site and urinary bladder are masked.

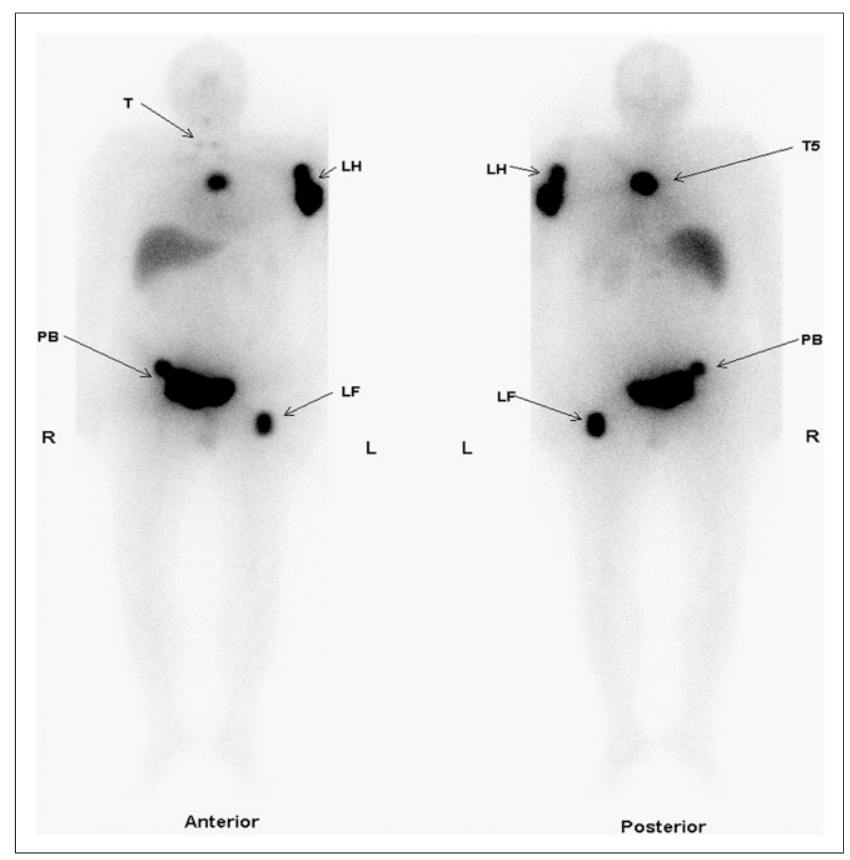

FIGURE 4. Postablation ${ }^{131} \mid$ whole-body scans showing residual uptake in thyroid bed $(T)$ and striking activity in left humerus (LH), thoracic spine (T5), right and left pelvic bones (PB), and left femur (LF).

that suggestion, an interesting alternative to avoid the stunning phenomenon when such a diagnosis is suspected may be to perform whole-body ${ }^{99 \mathrm{~m}} \mathrm{Tc}$-pertechnetate scanning as part of the staging process. The overall survival of patients with functioning metastatic thyroid cancer treated by radioiodine seems to be better than that of euthyroid patients (1).

\section{CONCLUSION}

This case is pathologically interesting in that papillary carcinoma metastatic to bone was able to produce hyperthyroidism. The scintigraphic features and outcome were also interesting in that there was preoperative uptake of ${ }^{99 \mathrm{~m}} \mathrm{Tc}$-pertechnetate despite the low TSH level and the improvement in myelopathic symptoms and hyperthyroidism after radioiodine therapy.

\section{DISCLOSURE}

No potential conflict of interest relevant to this article was reported.

\section{REFERENCES}

1. Hindié E, Mellière D, Lange F, et al. Functioning pulmonary metastases of thyroid cancer: does radioiodine influence the prognosis? Eur J Nucl Med Mol Imaging. 2003;30:974-981

2. Haq M, Hyer S, Flux G, Cook G, Harmer C. Differentiated thyroid cancer presenting with thyrotoxicosis due to functioning metastases. Br J Radiol. 2007;80:e38-e43.

3. Roodman GD. Mechanisms of bone metastasis. N Engl J Med. 2004;350:1655-1664.

4. Chantadisai M, Kingpetch K. Usefulness of ${ }^{99 \mathrm{~m}}$ Tc-pertechnetate whole body scan with neck and chest SPECT/CT for detection of post-surgical thyroid remnant and metastasis in differentiated thyroid cancer patients. Ann Nucl Med. 2014;28:674-682. 